\title{
Improved Empirical Modal Decomposition Coupled with Interwoven Fourier Decomposition for Building Vibration Signal Denoising
}

\author{
Xi Luo' ${ }^{1}$ and Shitu Abubakar $\mathbb{D}^{2}$ \\ ${ }^{1}$ School of Architecture and Art, Central South University, Changsha 410083, China \\ ${ }^{2}$ Department of Mechanical Engineering, Ahmadu Bello University, Zaria 810107, Nigeria \\ Correspondence should be addressed to Shitu Abubakar; abubakarshitu88@gmail.com
}

Received 28 December 2021; Accepted 22 January 2022; Published 10 February 2022

Academic Editor: Jiaqiang E

Copyright (C) 2022 Xi Luo and Shitu Abubakar. This is an open access article distributed under the Creative Commons Attribution License, which permits unrestricted use, distribution, and reproduction in any medium, provided the original work is properly cited.

\begin{abstract}
The precise detection of building vibration signals is a crucial problem for the identification of building vibration sources and characteristics. However, the building vibration signal is usually accompanied by complex high-frequency noise. The present study proposed a novel building vibration signal denoising method based on improved empirical modal decomposition coupled with interwoven Fourier decomposition (IEMD-IWFD). The noise-embed building vibration signal is first decomposed by the IEMD-IWFD. Then, the intrinsic mode function (IMF) components with useful information are extracted from the original building vibration signal using the energy criterion of the autocorrelation function. After that, the building vibration signal is formed by reconstructing the IMF component using the Hilbert transform. Based on the comparison of similarity coefficient and mean square error between the reconstructed signal from IEMD-IWFDM and EMD and target signal, it is indicated that the IEMD-IWFDM exhibits a better denoising performance for the simulated building vibration signal induced by trains.
\end{abstract}

\section{Introduction}

Building vibration signal processing is an indispensable part of vibration source identification and feature extraction. Poston et al. [1] reported on measurements from an instrumented, public building and examined the viability of conventional localization algorithms for locating persons moving within a building. Field measurements of vibration and noise on the ground and inside a nearby 3-story building subjected to moving subway trains were conducted by Zou et al. [2]. A general synthesis of identification and vibration control of building structures under unknown excitations was proposed by Lei et al. [3], which has been proven to be cost-effective and beneficial for developing smart building structures.

The key role of signal processing is to extract the desired section of the signal and remove the high-frequency noise. Some time, frequency and time-frequency domain-based methods have been proposed to suppress interference during vibration signal collection [4]. Traditional averaging approaches in the time domain are more suited to periodic signals [5]. Their denoising performance is limited to vibration signals with varying frequency and amplitude. Frequency domain methods such as Butterworth filtering and homomorphic filtering provide a better insight into the stability of a system, which can remove noise within the frequency band of interest [6]. The model frequency and its changing range caused by the variation of working conditions and surroundings are required when using frequency domain methods. Therefore, their application is limited [7]. Since characteristics such as time and frequency are taken into account, wavelet transform and other time-frequency domain techniques can be used on both static and nonstatic signals [8]. Nonetheless, some proper thresholds and optimal parameters should be manually selected and adjusted to avoid the elimination of useful information [9]. Other 
denoising methods based on global projection [10], artificial neural network [11], and singular value decomposition [12] have also been proposed to carry out the denoising tasks. Among the methods mentioned above for denoising vibration signals, the denoising performance depends on the selection and optimization of key parameters, which heavily rely on the researchers' empirical experiences.

Empirical mode decomposition (EMD) is an interesting and paramount direction in the field of signal processing. Lots of the works in the existing literature focus on nonlinear unsmooth signal analysis. For instance, a novel data-driven technique for the detection and isolation of faults was built upon pseudo-fault signal (PFS)-assisted EMD [13]. Ahn et al. [14] investigated fault detection of a roller bearing system using a wavelet denoising scheme and proper orthogonal values of an intrinsic mode function covariance matrix. Liu et al. [15] applied EMD-entropy to extract characteristic parameters from vibration signals of high voltage circuit breakers. EMD has been widely used in the engineering field owing to its adaptability. As the key point of the EMD, the screening process has a major influence on signal decomposition. The upper and lower envelopes, on the other hand, generated from cubic spline interpolation (CSI) in the EMD always lead to undershoots [16], overshoots [17], and edge effects [18], hence yielding unsatisfactory results. Li et al. [19] proposed an improved empirical modal decomposition (IEMD) algorithm. The latter is based on the modification of the envelope algorithm by repacking the CSI with $\mathrm{C} 2$ piecewise rational cubic spline interpolation (PRCSI) and C2 monotonic piecewise rational cubic spline interpolation (MPRCSI), which presented the superiority, especially for those nonlinear and unstable signals. However, the IEMD algorithm is not good when decomposing those signals with the overlapped spectra [20]. As an auxiliary measure to the IEMD, interwoven Fourier decomposition (IWFD) can extract signals by optimizing bandwidths in the frequency domain. The IWFD is a digital information timefrequency analytical technique that is adaptable. Quasibandpass filters with zero phase are used to decompose the signal into a few Fourier intrinsic band functions guaranteeing the signal's complete rebuilding in the IWFD [21].

As mentioned above, although extensive investigations have been conducted in the field of building vibration signals, research report in the denoising of building vibration signals, which remains a challenging task, is limited. Moreover, it becomes more difficult when signals disturbed by noise have nonlinear and nonsteady characteristics. Therefore, the authors of this study evaluated the denoising performance for the building vibration signal using the IEMD coupled with the IWFD (referred to as IEMD-IWFD).

\section{Strategy for Building Vibration Signal Denoising}

Figure 1 shows the procedure flowchart for building vibration signal denoising. The IEMD-IWFD is employed to decompose the real building vibration signal into intrinsic mode functions (IMFs). After that, the IMFs are extracted using the energy criterion of the autocorrelation function (ACF). After the determination of IMFs, the target building vibration signal is formed by IMFs reconstruction by the Hilbert transform. The details of the algorithms used in the current denoising method are described as follows.

\subsection{Description of IEMD-IWFD}

2.1.1. IEMD Algorithm. Many researchers have employed the EMD method, which is an adaptive algorithm to perform the reconstruction [22], classification [23], and denoising [24] of a signal. However, the use of CSI to generate peak and lowest envelopes has some disadvantages such as overtones and subtones. EMD encountered difficulties in fitting signals with the local extremum of CSI. Some methods have been proposed to improve the adaptability of cubic spline [25]. One of these methods is the Hermite cubic spline with a shape controlling parameter developed by Li et al. [19]. The shape controlling parameter is more flexible than the CSI, but it still cannot satisfy the flexibility and smoothness simultaneously. There has been lots of research on the C2 cubic spline, which can interpolate the monotone, positive, and convex data [25].

For a given dataset $((x i, f i), i=0,1, \ldots, n)$ with $x 0<x 1<\ldots<x n$, let $h i=x i+1-x i, \Delta i=(f i+1-\mathrm{fi}) / \mathrm{hi}$ and $\theta=(x-x i) / h i \quad(0<\theta<1)$. A well-advised cubic spline for interpolating $I i=[x i, x i+1]$ is defined as follows:

$$
S_{i}(x)=\frac{M_{i}(x)}{N_{i}(x)}=\frac{P_{i 0}(1-\theta)^{3}+P_{i 1} \theta(1-\theta)^{2}+P_{i 2} \theta^{2}(1-\theta)+P_{i 3} \theta^{3}}{(1-\theta)^{2} \alpha_{i}+\theta(1-\theta)\left(2 \alpha_{i} \beta_{i}+\gamma_{i}\right)+\theta^{2} \beta_{i}}
$$

An unknown $P_{i k}(k=0,1, \ldots, n)$ has the following form:

$$
\left\{\begin{array}{l}
P_{i 0}=\alpha_{i} f_{i}, \\
P_{i 1}=\left(2 \alpha_{i} \beta_{i}+\alpha_{i}+\gamma_{i}\right) f_{i}+\alpha_{i} h_{i} d_{i}, \\
P_{i 2}=\left(2 \alpha_{i} \beta_{i}+\beta_{i}+\gamma_{i}\right) f_{i+1}-\beta_{i} h_{i} d_{i}, \\
P_{i 3}=\beta_{i} f_{i+1},
\end{array}\right.
$$

where the parameters $\alpha_{i}>0, \beta_{\mathrm{i}}>0$, and $\gamma_{i} \geq 0$ are used to adjust the interpolation curve shape. For instance, the data range, monotonicity, positivity, and convexity over the complete gap can be controlled. Based on the C2 continuity, the following linear system can be described:

$$
a_{i} d_{i-1}+b_{i} d_{i}+c_{i} d_{i+1}=e_{i}, i=1,2, \ldots, n-1,
$$




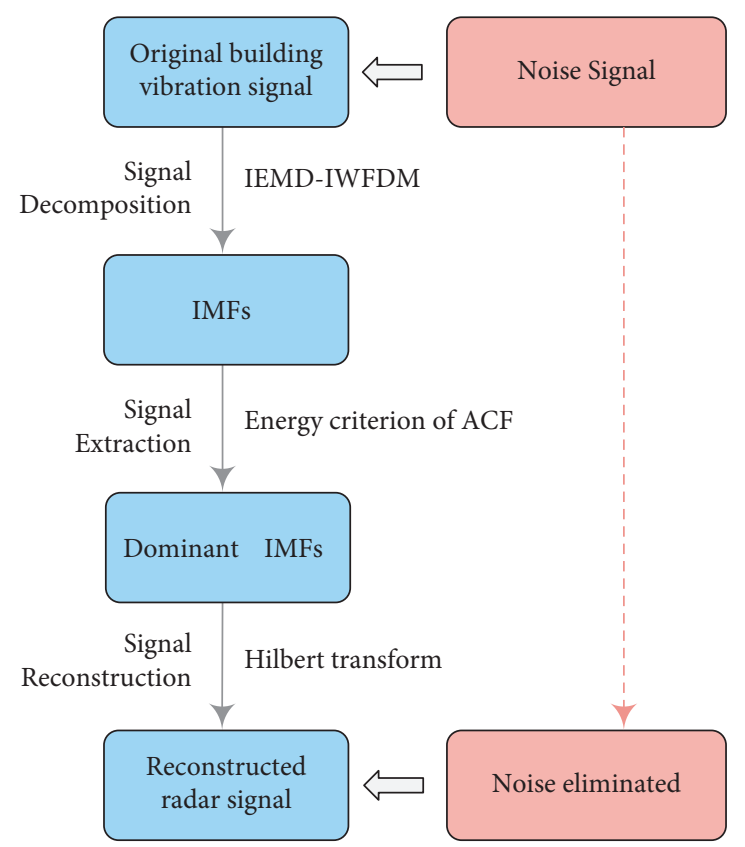

Figure 1: Procedure flowchart of building vibration signal denoising.

where

$$
\left\{\begin{array}{l}
a_{i}=h_{i} \alpha_{i-1} \alpha_{i}, \\
b_{i}=h_{i} \alpha_{i}\left(\gamma_{i-1}+2 \alpha_{i-1} \beta_{i-1}\right)+h_{i-1} \beta_{i-1}\left(\gamma_{i}+2 \alpha_{i} \beta_{i}\right), \\
c_{i}=h_{i-1} \beta_{i-1} \beta_{i}, \\
e_{i}=h_{i} \alpha_{i}\left(\gamma_{i-1}+\alpha_{i-1}+2 \alpha_{i-1} \beta_{i-1}\right) \Delta_{i-1}+h_{i-1} \beta_{i-1}\left(\gamma_{i}+\beta_{i}+2 \alpha_{i} \beta_{i}\right) \Delta_{i},
\end{array}\right.
$$

Clearly, the first derivative variable $d_{i}$ can be calculated with good approximation based on the aforementioned linear system and coupled with two more equations such as $s(t) \quad(x 0)=d 0$ and $s(t) \quad(x n)=d n$, and the first derivative parameter $d_{i}$ can be calculated through a unique solution from this linear system. Two options for end-point derivatives were explored by Karim and Pang [25], $d_{0}$ and $d_{n}$. There is only one option in this case, which takes into account the equation.

$$
d_{0}=\Delta_{0}+\left(\Delta_{0}-\Delta_{1}\right)\left(\frac{h_{0}}{h_{0}+h_{1}}\right) \text { and } d_{n}=\Delta_{n-1}+\left(\Delta_{n-1}-\Delta_{n-2}\right)\left(\frac{h_{n-1}}{h_{n-1}+h_{n-2}}\right) \text {. }
$$

For the choice of the parameters, they have tested some cases to guarantee the smoothness of curves with different interpolation shapes, and the C2 PRCSI has been extensively validated. In the comparison between the simulated results from $[25,26]$, it is proved that the performance of the $\mathrm{C} 2$ cubic spline performed well. Nevertheless, there is no useful information mentioned for the monotonicity, and thus, a monotone version for the C2 PRCSI will be further introduced.
Abbas et al. [26] investigated the shape-preserving C2 PRCSI for monotone data. It can be seen that it appears with the original form of C2 PRCSI at every span $I i=[x i$, $x i+1]$.

$$
S_{i}(x)=\frac{P_{i}(\theta)}{Q_{i}(\theta)}
$$

where 


$$
\begin{aligned}
& P_{i}(\theta)=\alpha_{i} f_{i}(1-\theta)^{3}+\left(f_{i}\left(2 \alpha_{i}+\beta_{i}+\gamma_{i}\right)+\alpha_{i} h_{i} d_{i}\right) \theta(1-\theta)^{2}+\left(f_{i+1}\left(\alpha_{i}+2 \beta_{i}+\gamma_{i}\right)-\beta_{i} h_{i} d_{i+1}\right) \theta^{2}(1-\theta)+\beta_{i} f_{i+1} \theta^{3}, \\
& Q_{i}(\theta)=(1-\theta)^{2} \alpha_{i}+\theta(1-\theta)\left(\gamma_{i}+\alpha_{i}+\beta_{i}\right)+\theta^{2} \beta_{i} .
\end{aligned}
$$

At the same boundary conditions as mentioned above, such as the variables that keep the shape of $\alpha_{i}>0, \beta_{i}>0$, $\gamma_{i} \geq 0$ and the dataset $((x i, f i), i=0,1, \ldots, n)$ with $x i<x i+1$ and $f i<f i+1$, etc., two cases for the monotonical series are presented as follows.
Case 1. $\Delta_{i}=0, \quad d_{i}=d_{i+1}=0 \quad$ and $\quad S_{i}(x)=f_{i}, \forall x \in I_{i}$, $i=0,1, \ldots, n-1$;

Case 2. $\Delta_{i}>0, \quad S_{i}^{(1)}(x)=\sum_{k=0}^{4}(1-\theta)^{4-k} \theta^{k} M_{k i} /\left(q_{i}(\theta)\right)^{2}$, where

$$
\left\{\begin{array}{l}
M_{0 i}=\alpha_{i}^{2} d_{i} \\
M_{1 i}=2 \alpha_{i}\left(\left(\alpha_{i}+2 \beta_{i}+\gamma_{i}\right) \Delta_{i}-\beta_{i} d_{i+1}\right) \\
M_{2 i}=M_{1 i}+M_{3 i}-\left(M_{0 i}+M_{4 i}\right)+\gamma_{i}\left(\alpha_{i}+\beta_{i}+\gamma_{i}\right) \Delta_{i}-2 \alpha_{i} \beta_{i}\left(d_{i}+d_{i+1}\right) \\
M_{3 i}=2 \beta_{i}\left(\left(2 \alpha_{i}+\beta_{i}+\gamma_{i}\right) \Delta_{i}-\alpha_{i} d_{i}\right) \\
M_{4 i}=\beta_{i}^{2} d_{i+1}
\end{array}\right.
$$

The C2 curve for monotone data is preserved by the rational cubic function when the shape parameters meet the following requirements:

$$
\left\{\begin{array}{l}
\alpha_{i}>0, \\
\beta_{i}>0 \\
\gamma_{i}=m_{i}+\max \left\{0, \frac{\left(d_{i+1} \beta_{i}-\Delta_{i}\left(\alpha_{i}+2 \beta_{i}\right)\right)}{\Delta_{i}}, \frac{\left(d_{i} \alpha_{i}-\Delta_{i}\left(2 \alpha_{i}+\beta_{i}\right)\right)}{\Delta_{i}}\right\}
\end{array}\right.
$$

where $m_{i}$ is a constant larger than 0 ,

The interpolation error was investigated in [18], whereby the minimum interpolation error is lower than 0.064 . Compared to other alternative approaches, it is very diminutive, and this implies that the C2 MPRCSI with the parameter choice schemes presented in [26] is computationally economical and pictorially appealing in comparison with the local and global schemes [27] and the scheme developed in Ref. [28].

2.1.2. IWFD Algorithm. The Fourier decomposition algorithm with adaptability characteristics is an adaptive datadriven signal decomposition technique that generates the signal's analytic illustration and Hilbert transform [29]. Compared to the EMD algorithm, the main advantage of the Fourier decomposition algorithm is that it has more detailed mathematical expressions. As a result, there is no need to predefine the parameters such as iterations, window lengths, and termination criteria. When using the Fourier decomposition algorithm, a signal can be considered as a series of zero-mean orthosecting functions known as analytical Fourier intrinsic band functions.

In the Fourier decomposition algorithm, a real-valued $\mathrm{N}$-point signal $\mathrm{x}[n]$ is represented by the weighted sum of Fourier bases based on the discrete Fourier transform (DFT):

$$
x[n]=\sum_{k=0}^{N-1} X[k] W_{N}^{-k n},
$$

where $W_{N}=e-j(2 \pi / \mathrm{N})$ and $x[k]$ denotes the $N$-point DFT coefficients of the signal $x[n]$. These coefficients can be achieved in a numerically effective way using fast Fourier transform (FFT) techniques.

The $\mathrm{N}$-point signal $x[n]$ can be rewritten by the following two forms: 


$$
\begin{aligned}
& x[n]=X[0]+\sum_{k=1}^{N / 2-1} X[k] W_{N}^{-k n}+X\left[\frac{N}{2}\right] W_{N}^{-N n / 2}+\sum_{k=N / 2+1}^{N-1} X[k] W_{N}^{-k n}, \\
& x[n]=X[0]+\sum_{k=1}^{(N-1) / 2} X[k] W_{N}^{-k n}+\sum_{k=(N-1) / 2+1}^{N-1} X[k] W_{N}^{-k n} .
\end{aligned}
$$

Due to $\mathrm{x}(n)$ being a real-valued signal, $\sum_{k=1}^{N / 2-1} X[k] W_{N}^{-k n}$ and $\sum_{k=N / 2+1}^{N-1} X[k] W_{N}^{-k n}$ are a pair of complex conjugates. The same holds true for $\sum_{k=1}^{(N-1) / 2} X[k] W_{N}^{-k v}$ and $\sum_{k=(N-1) / 2+1}^{N-1} X[k] W_{N}^{-k}$ in equation (12). Two definitions are made as follows:

$$
\begin{aligned}
& z[n] \triangleq 2 \sum_{k=1}^{N / 2-1} X[k] W_{N}^{-k n}, \\
& v[n] \triangleq 2 \sum_{k=1}^{(N-1) / 2} X[k] W_{N}^{-k n} .
\end{aligned}
$$

The N-point signal $x[n]$ can be expressed in a simpler form as

$$
x[n]= \begin{cases}X[0]+\operatorname{Re}\{z[n]\}+(-1)^{n} X[N / 2], & N: \text { even }, \\ X[0]+\operatorname{Re}\{v[n]\}, & N: \text { odd }\end{cases}
$$

where the real parts of $z[n]$ and $v[n]$ signals are stated by $\operatorname{Re}(z[n])$ and $\operatorname{Re}(v[n])$, respectively. To reduce the complexity in Fourier decomposition equations, signals with even values of $\mathrm{N}$ are only considered. The $z[n]$ signal is factorizable as in

$$
2 \sum_{k=1}^{N / 2-1} X[k] W_{N}^{-k v}=\sum_{i=1}^{M} a_{i}[n] e^{j \phi_{i}[n]}
$$

where $\varphi_{i}[n]$ and $a i[n]$ are the real-time scenario and instantaneous frequency, respectively, and $M$ is the total number.

Equation (16) expressed the decomposition process which can be performed under the following conditions for each analytic Fourier intrinsic band function:

$$
\begin{aligned}
& f_{i}[n]=\frac{1}{2 \pi}\left(\phi_{i}[n+1]-\phi_{i}[n]\right) \geq 0, \\
& a_{i}[n] \geq 0, n=0,1,2, \ldots \ldots N-1,
\end{aligned}
$$

where $f_{i}[\mathrm{n}]$ is the instantaneous frequency of the analytic Fourier intrinsic band function.

In a forward search, i.e., the scanning from low to high frequency, the decomposition process in equation (17) is carried out by [29]

$$
a_{i}[n] e^{j \phi_{i}[n]}=2 \sum_{k=N_{i}-1+1}^{N_{i}} X[k] W_{N}^{-k n}
$$

where $N_{0}=0$ and $N_{M}=N / 2-1$. In a backward search, i.e., the scanning from high to low frequency, the decomposition process in equation (17) is carried out by

$$
a_{i}[n] e^{j \phi[n]}=2 \sum_{k=N_{i}}^{N_{i-1}-1} X[k] W_{N}^{-k n} .
$$

The key points of (19) and (20) aim to confirm the frequency $N_{1}, N_{2}, \ldots, N_{M-1}$. When the $N_{i}$ is used, the righthand side of equation (15) and its corresponding instantaneous phase and instantaneous frequencies are calculated for $n=0,1, \ldots, N-1$. The minimum amount of possible $N_{i}$ for all $n$ is determined by the frequency index $N_{i}$.

2.1.3. IEMD Coupled with IWFD. The envelope algorithm based on $\mathrm{C} 2$ piecewise rational cubic spline interpolation (PRCSI) and C2 monotone piecewise rational cubic spline interpolation (MPRCSI) was employed to decompose the building vibration signal corrupted by noise. The zero-phase nonrectangular bandpass filters contained in the IWFD were coupled into the IEMD to analyze signals with multiple components and intersecting instantaneous frequencies. We first structured the upper envelope and lower envelope using the C2-PRCSI method. Considering the presence of undershoots, we further iteratively modified the original envelope using C2 MPRCSI technology to eliminate undershoots as accurately as possible. With the assistance of IWFD, filter parameters were evaluated in order to ensure that the sum of all Fourier intrinsic band functions can reconstruct the entire signal, which effectively improves the compatibility of multicomponent building vibration signal processing. Figure 2 presents the construction flowchart of the IEMD-IWFD method. As it can be noticed and based on the traditional EMD method, modified cubic spline interpolations are inserted to optimize the envelopes of the original building vibration signal. This is done so that a relatively high accuracy and efficiency decomposition for the extremely nonlinear and nonstationary building vibration signals is achieved. The determination of the IFM signal refers to the decomposition process of the original signals in IWFD. In a bid to analyze the signals of intersecting instantaneous frequencies, the zero-phase nonrectangular bandpass filters referred from IWFD were employed to assist the IEMD method. The features of these filters are determinate, and as a result, the sum of all FIBFs ensures a full rebuilding of the signal. When compared with EMD, the IEMD-IWFD is expected to be more competitive for the 


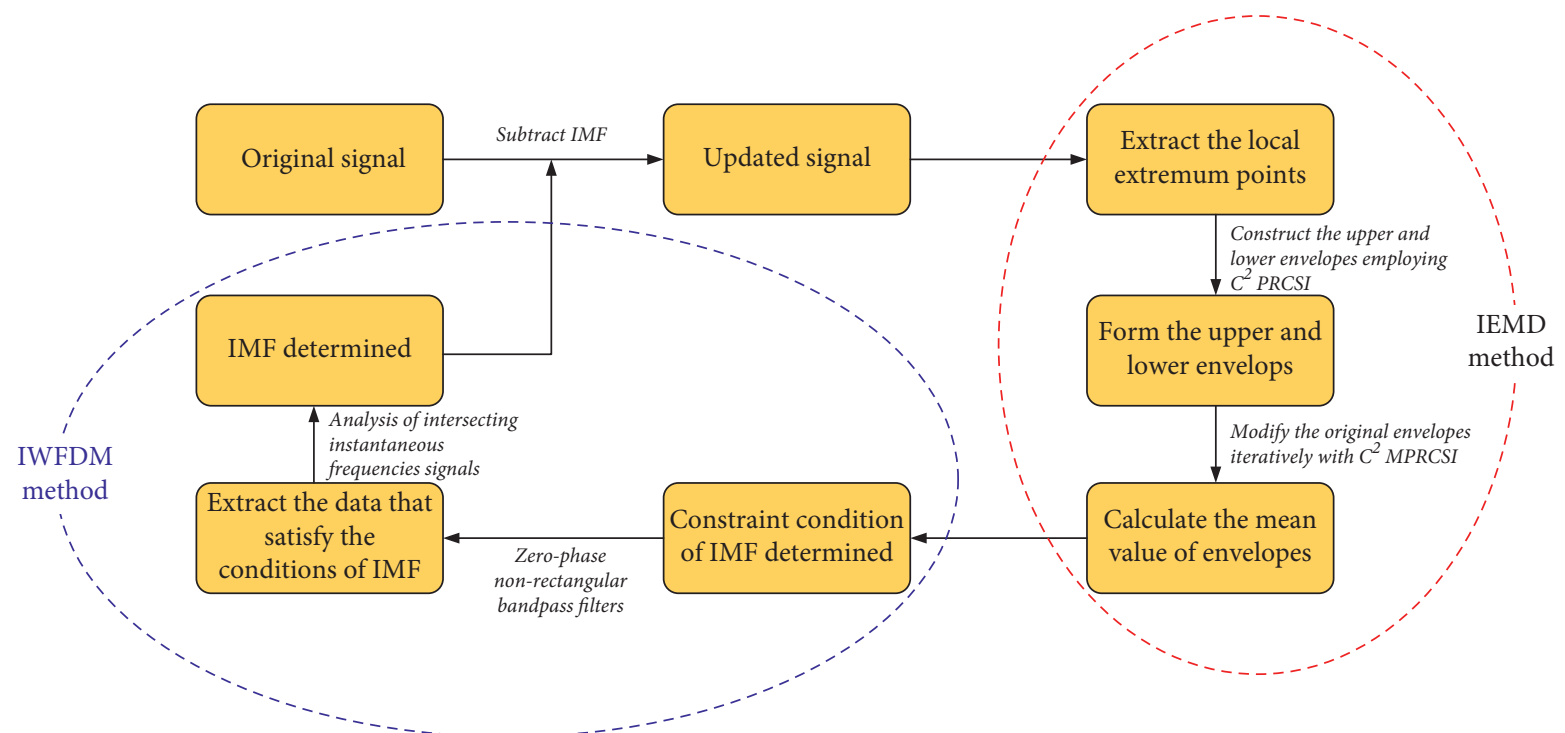

Figure 2: Procedure of IEMD-IWFD construction.

denoising of nonlinear and nonstationary building vibration signals and more compatible for analyzing the intersecting instantaneous frequencies of building vibration signals.

2.2. Description of Energy Criterion of ACF. The correlation function is an average measure of signal time-domain characteristics, which describes the similarity degree between signals at different times. The time average-based ACF is calculated as follows:

$$
R_{x}(m)=\frac{1}{N-|m|} \sum_{n=0}^{N-1 \dashv \tau \mid} x_{N}(n) x_{N}(n+m),
$$

where $m$ represents the time delay. In order to ensure the consistency between the sequence length of ACF and the length of signal $x N(n)$, the time delay $m$ should satisfy $(-N /$ $2)-1<m<N / 2$. This sequence energy is expressed as follows:

$$
E(n)=\sum_{n=0}^{N-1} x^{2}(n)
$$

The ACF of each component of the decomposed signal can be calculated, and the distribution characteristics of the ACF can be obtained. Due to weak correlation and strong randomness at each moment of the random noise, the ACF reaches its maximum value at the zero point, while the value of the ACF rapidly decreases to a small value at other points. Since there is a correlation between general signals at different times, the autocorrelation function value outside of zero cannot quickly decay to very small values, but changes with the varying time difference, especially for the periodic signal. The ACF is still a periodic signal and its change law is different from the changing rule of the noise ACF obviously. The dominant IMFs can be determined according to the ACF characteristics of each component.
2.3. Description of Signal Reconstruction Based on Hilbert Transform. The IMFs of the original signal can be regarded as a set of multidimensional components closely related to the information of the source signal. By calculating the correlation between time-frequency characteristics, the original signal can be recovered from these components. Based on the signal decomposition, the basic flowchart of target signal recovery is shown in Figure 3. The Hilbert transformation is used in multichannel IMFs to solve the instantaneous frequency of signal components and obtain the time-frequency characteristics of the signal. The noise and/or invalid signals can be filtered out based on the timefrequency distribution of signal components. Based on IMF' $[n]$ and coefficient matrix $A$, the source signal $S$ in the mixed signal is separated, which can be expressed as

$$
\mathrm{A} \cdot \operatorname{IMF}[n]=\mathrm{S} \text {. }
$$

To explore the time-frequency behavior of each IMF component, the Hilbert spectrum analysis was used in this study to process the signals. Unlike FFT time-frequency analysis, the Hilbert spectrum analysis is performed without using the basic variable frequency to represent the behavior of the signal. It analyzes the signal from the perspective of instantaneous frequency and expands the signal into a timefrequency plane. The Hilbert spectrum analysis is derived by applying Hilbert transform to any signal $x(t), t \in(-\infty,+\infty)$, stated as

$$
y(t)=H[x(t)]=\int_{-\infty}^{+\infty} \frac{x(\tau)}{\pi(t-\tau)} d \tau .
$$

Due to the integral singularity $\tau=t$, the formula expressed by Cauchy principal value integrals is given as

$$
y(t)=H[x(t)]=\frac{1}{\pi} \mathrm{P} . \mathrm{V} \int_{-\infty}^{+\infty} \frac{x(\tau)}{t-\tau} d \tau,
$$




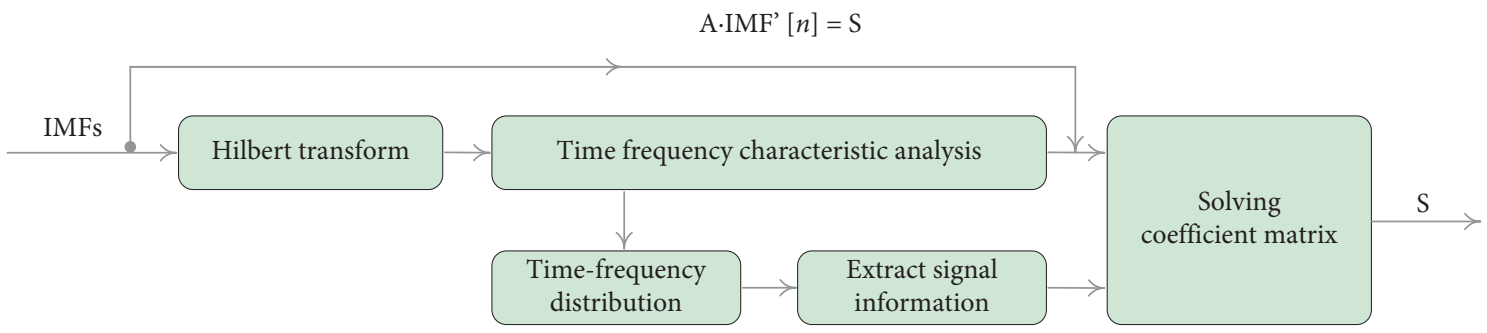

Figure 3: Procedure of target signal recovery.

where P.V represents the generalized Cauchy principal valued integral.

Considering $y(t)$ is the convolution of signal $x(t)$ and $1 /$ $\pi t$, the above relationship also exists as

$$
y(t)=x(t) \cdot \frac{1}{t \pi} .
$$

It is known by the property of Fourier transform that

$$
\begin{gathered}
F(y)=F[1 / t \pi] F[x(t)], \\
F[1 / t \pi]=-j \operatorname{sgn}(f)=\left\{\begin{array}{l}
-j, f>0 \\
j, f<0
\end{array}=\left\{\begin{array}{l}
e^{-j \frac{\pi}{2}}, f>0 . \\
\pi \\
e^{j \frac{\pi}{2}}, f<0 .
\end{array}\right.\right.
\end{gathered}
$$

To understand the meaning of the Hilbert transform, expressed as type

$$
z(t)=x(t)+j y(t)=a(t) e^{j \theta(t)},
$$

where $a(t)=(x 2+y 2) 1 / 2$ is the amplitude of the signal and $\theta(t)=\tan ^{-1}(y / x)$ is the instantaneous response signal.

The frequency at any instance is expressed as

$$
\omega(t)=\frac{d \theta(t)}{d t} .
$$

The instantaneous frequency of the signal represents the aggregation degree of signal energy in a certain frequency band. Based on the Hilbert transform on IMF components, the original signal is given as

$$
x(\omega, t)=\sum_{j=1}^{n} a_{j}(t) \exp \left(i \int \omega_{j} d t\right) .
$$

After the transformation in the above steps, the amplitude and frequency of the original signal are given as a function of time. If the signal is represented on the timefrequency-energy three-dimensional distribution, the Hilbert spectrum $H(\omega, t)$ of the signal can be obtained as

$$
H(\omega, t)=\sum H_{i}(\omega, t) .
$$

\section{Results and Discussion}

A model was proposed by Rossi and Nicolini [30] to predict the building vibration induced by trains, as shown in equation (3), in which the train's quality and velocity, the rail's displacement, the soil's characteristics, and the sleeper's spacing were considered as

$$
\begin{aligned}
P_{\max } & =\int_{-T / 2}^{T / 2} \frac{M g s v_{t} K e^{-\alpha \sqrt{x^{2}+d^{2}}}}{T 2 \pi i \sqrt{x^{2}+d^{2}}} d x, \\
L_{v} & =20 \log \left(\frac{\sqrt{P_{\max } / \rho_{s} c_{R}}}{v_{\text {ref }}}\right),
\end{aligned}
$$

where $P_{\max }$ is the largest time-average power, $M$ the total train mass, $s$ the longest vertical rail displacement, vt the velocity of train, $K$ the constant depended on model calibration, $\alpha$ the dissipation constant of soil, $d$ the distance between power and train, $T$ the length of train, $i$ the spacing between adjoining sleepers, $\mathrm{L}_{\mathrm{v}}$ the level of vibration, $\rho_{s}$ the surface density of soil, and $c_{R}$ the velocity of wave's propagation, and $\mathrm{v}_{\text {ref }}$ equals $2.54 \times 10-8 \mathrm{~m} / \mathrm{s}$.

Developing a reliable prediction model for noise is necessary to prevent the interference of environmental noise. Some noise prediction models have been developed and codified into industrial standards based on test conditions and industrial circumstances. A model was proposed by Nassiri et al. [31] to assess the noise induced by the train, which is related to the velocity of the train, vt, and the distance between power and train $d$, as shown in equation (35). However, the impact of the curve, joint, and turnout of the train were not considered, and thus, this model can only be used to predict the noise caused in the testing line.

$$
L_{\mathrm{n}}=L_{0}-K_{1} \lg \left(\frac{d}{d_{0}}\right)+K_{s} \lg \left(\frac{v}{v_{0}}\right),
$$

where $L_{n}$ is the equivalent sound level, $K_{l}$ and $K_{s}$ are the correction factors of length and speed, and $L_{0}, d_{0}$, and $v_{0}$ are the reference values of sound level, distance, and speed, respectively.

The simulated building vibration signal without and with the noise are given in Figures 4(a) and 4(b), respectively.

The denoising of building vibration signals requires signal decomposition first. Figure 5 shows the decomposed IMF components based on the method of IEMD-IWFD. The synthetic building vibration signal is decomposed into 7 IMFs, which are ordered according to frequency. IMF1 and IMF2 are obviously high-frequency components, while IMF5-IMF7 are relatively low-frequency components.

The decomposed results of synthetic building vibration using the EMD are presented in Figure 6. As can be seen, two fewer IMF components are obtained from the EMD 


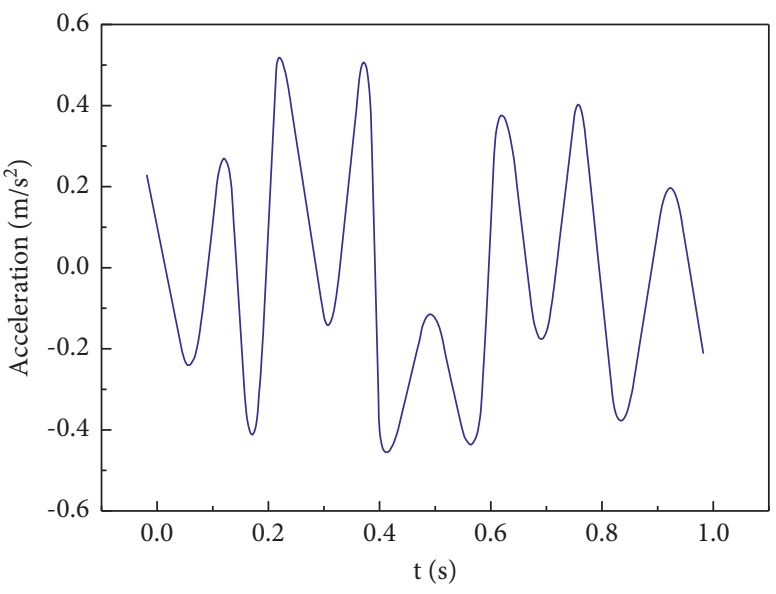

(a)

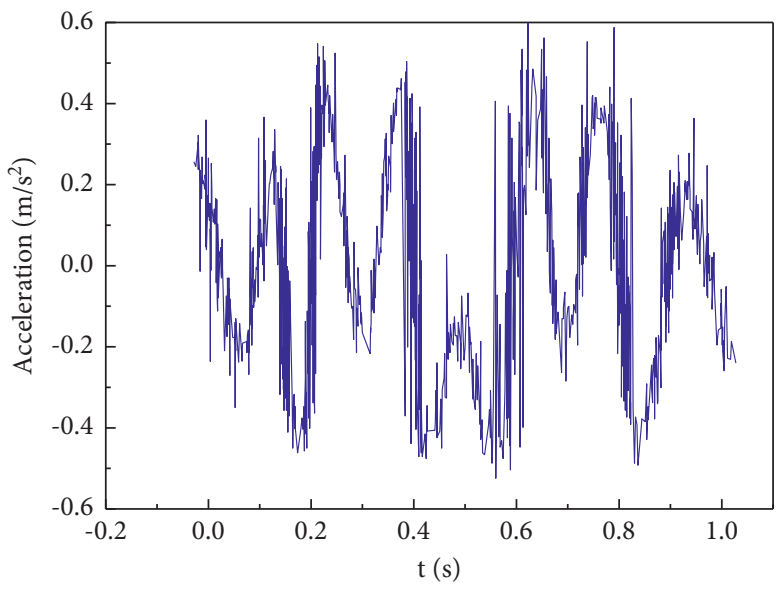

(b)

FIGURE 4: Simulated building vibration signal without and with noise.

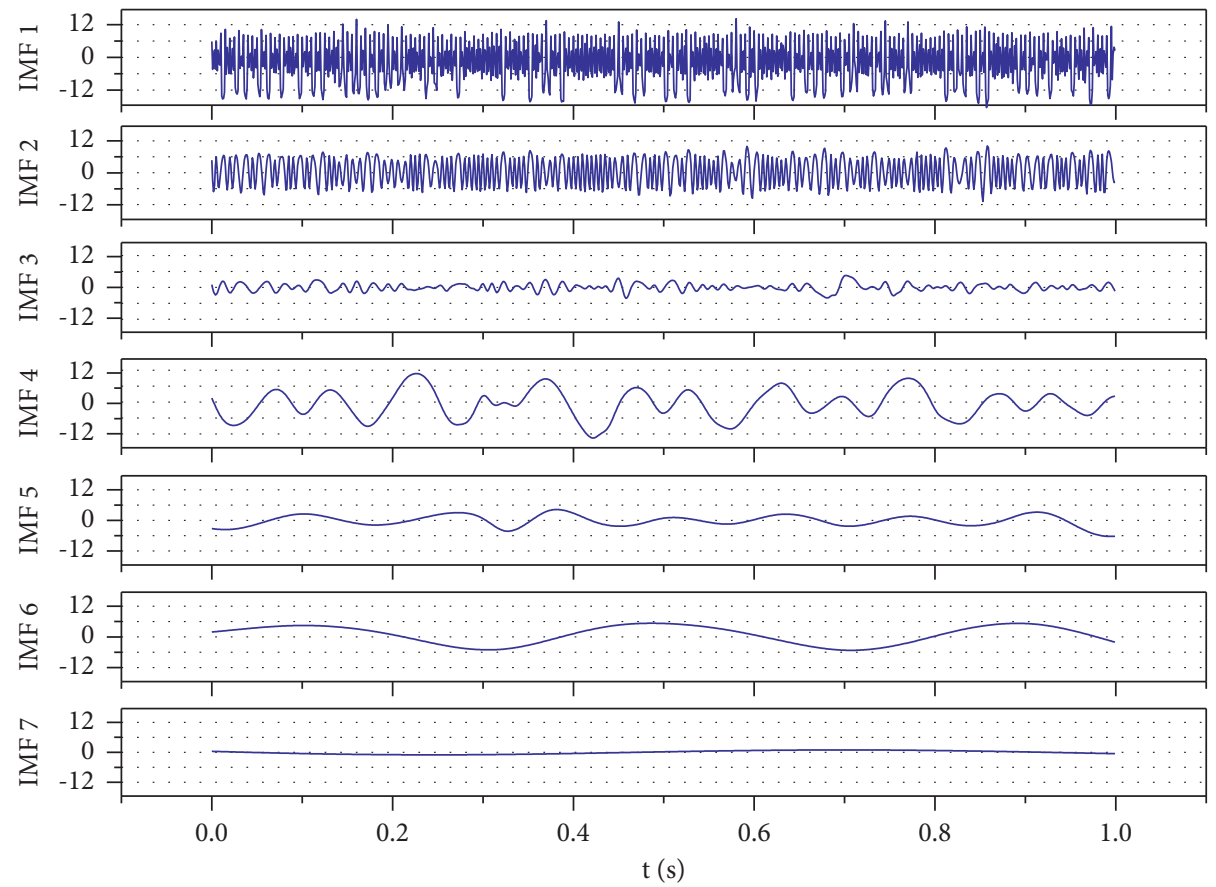

FIGURE 5: IMFs decomposed from building vibration signal using IEMD-IWFD.

compared to the IEMD-IWFD. And the frequency change in IMFs is distinct: high-frequency IMF components are mainly concentrated in IMF1, while IMF2 and IMF3 show relatively high-frequency signals.

Many experimental studies revealed many statistical characteristics of noise after decomposition. In fact, each IMF component complies with the normal distribution. After the signal mixed with noise is decomposed, the IMF component with a small ordinal number mainly contains the highest frequency part of the noise, while the IMF component with a large ordinal number mainly contains the lowest frequency part of the noise. The frequency-domain characteristic of noise is destroyed. The noise in each IMF component is no longer the real noise, but still has the statistical features of noise. With the similar distribution of the general noise autocorrelation function, the autocorrelation function of the noise in each IMF component reaches its maximum value at zero and rapidly decays to zero at other points.

Figure 7 shows the autocorrelation function calculated for the decomposed IMFs by IEMD-IWFD. According to the distribution characteristics of the signal autocorrelation function, the high frequency part of the noise, useful information components, and low frequency part of the noise are mainly contained in IMF1-3, IMF4-5, and IMF6-7, respectively. The energy variation curve of the autocorrelation function calculated by IMFs obtained by EMD decomposition is shown in Figure 8. It can be seen that the 

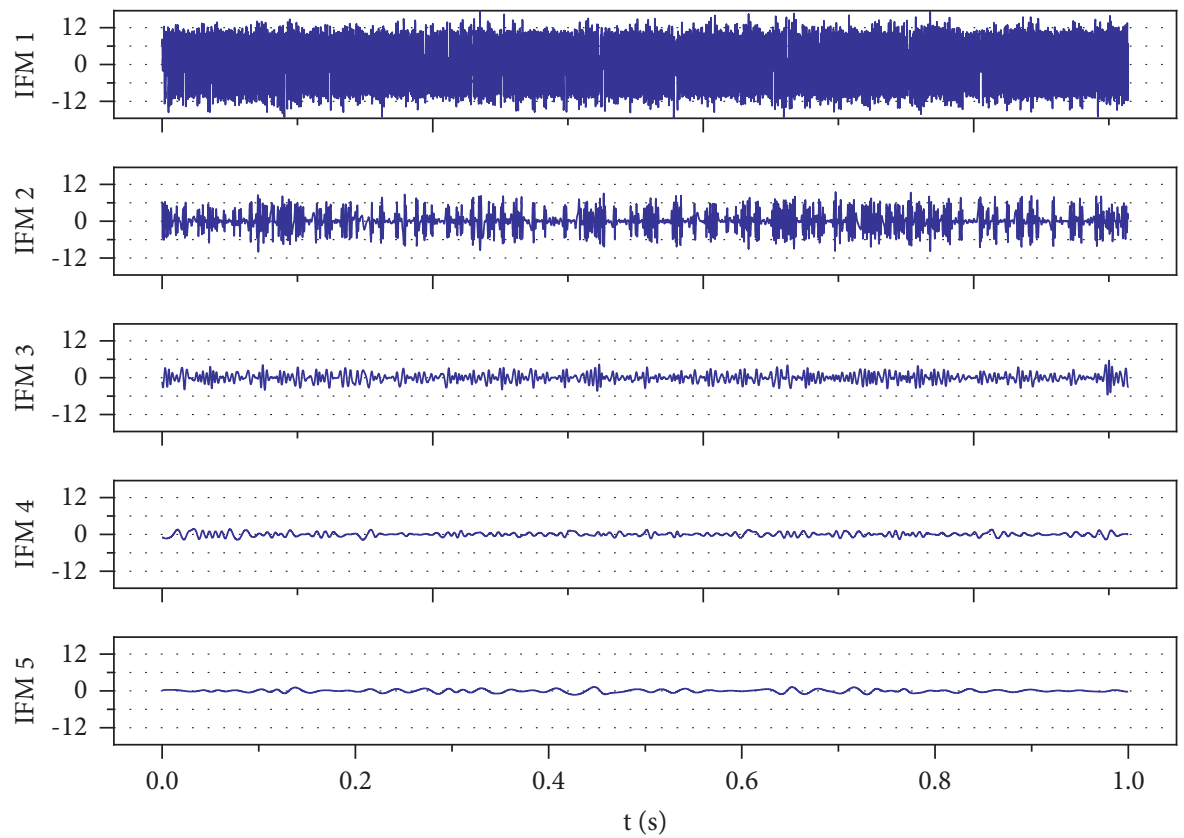

Figure 6: IMFs decomposed from building vibration signals using EMD.

autocorrelation function of IMF1-2 has low energy, while the autocorrelation function of IMF3-4 has high energy. Thus, it is indicated that the decomposed IMFs dominated by useful information by IEMD-IWFD and EMD are IMF45 and IMF3-4 components, respectively.

After obtaining the IMF components dominated by useful information, their weight coefficients were obtained by solving the coefficient matrix based on the Hilbert transform, and the target signal was reconstructed as follows. The transformation process of signal extraction time-frequency characteristics is represented by $F[\cdot]$, and the relationship between the mixed signal and an IMF part is represented as

$$
\begin{aligned}
\mathrm{A} \cdot F[I M F] & =F[\widehat{S}], \\
F[I M F] & =\mathrm{A}^{-1} \cdot F[\widehat{S}],
\end{aligned}
$$

where $A$ is the coefficient matrix related to the time change and $\widehat{S}$ represents the time domain estimate of the source signal. By applying the Hilbert transform to both sides of the equation, the time-frequency relationship of the signal can be transformed into the relationship between time and energy, which can be expressed as

$$
H^{-1}[F[I M F]]=H^{-1}\left[A^{-1} \cdot F[\widehat{S}]\right] .
$$

In the process of using the time-frequency behavior of each IMF component to reconstruct the time-frequency characteristics of the source signal, the coefficient matrix A essentially represents the weight value of each component at each time node, i.e., the component with the larger A corresponds to the larger correlation with the reference value.

The reconstructed building vibration signal based on IEMD-IWFDM and EMD is shown in Figure 9. It is clear from Figure 9(a) that the reconstructed building vibration signal recovers well which benefits from the good separation of the synthetic building vibration signal. And the signal amplitude slightly changes after the reconstruction because the building vibration signal mainly uses frequency modulation, which leads to the amplitude changes having a relatively small impact on the separation performance. However, it is observable in Figure 9(b) that the noise interference was not exhaustively eliminated in the reconstructed building vibration signal, which indicates that the signal decomposition method of EMD has poorer performance on building vibration signal denoising compared with IEMD-IWFDM.

Figure 10 shows an experimental building vibration signal collected from the high-speed rail station of Changsha, in which the abscissa and ordinate represent frequency and sound level, respectively. The setup was built to maintain the working of train and staff. The vibration of buildings mainly comes from the running of trains. The noise from rails, aerodynamic, and machinery are the major noise sources.

Figure 11 shows the denoised building vibration signal by EMD and the IEMD-IWFD methods. The signal is reconstructed after removing the recognized high-frequency IMF component, which is regarded as a noise signal. As seen, the denoised signal by the conventional EMD method has a serious distortion. Especially at the distance from 0 to $1 \mathrm{~km}$, the signal cannot be effectively recovered. The denoised data from 1 to $6 \mathrm{~km}$ is similar to the original echo signal, but still has large fluctuations. The denoised result obtained by the IEMD-IWFD method is much better than the EMD method since the noise can be effectively separated and eliminated and the signal details can be kept to a certain extent. 


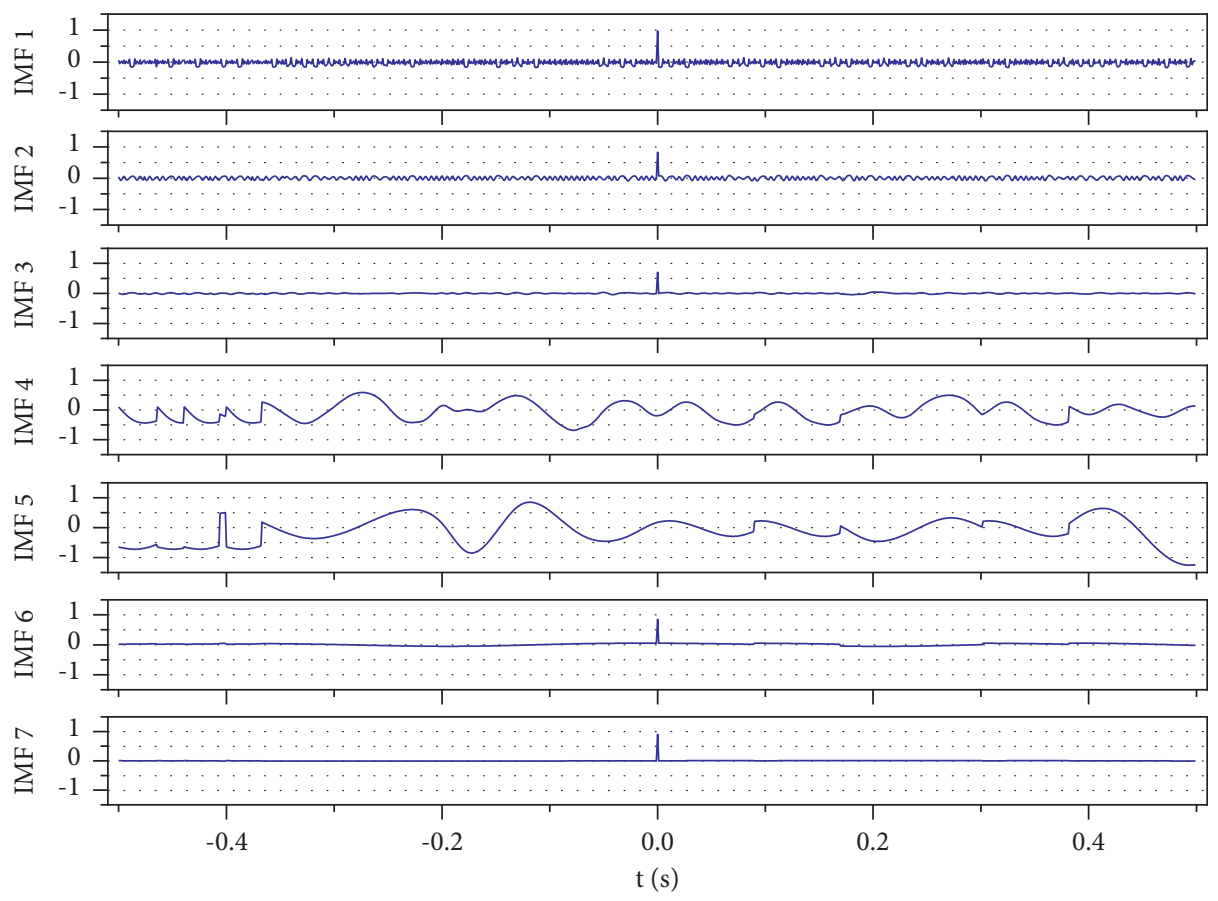

FIGURE 7: ACF of each IMF by IEMD-IWFD.

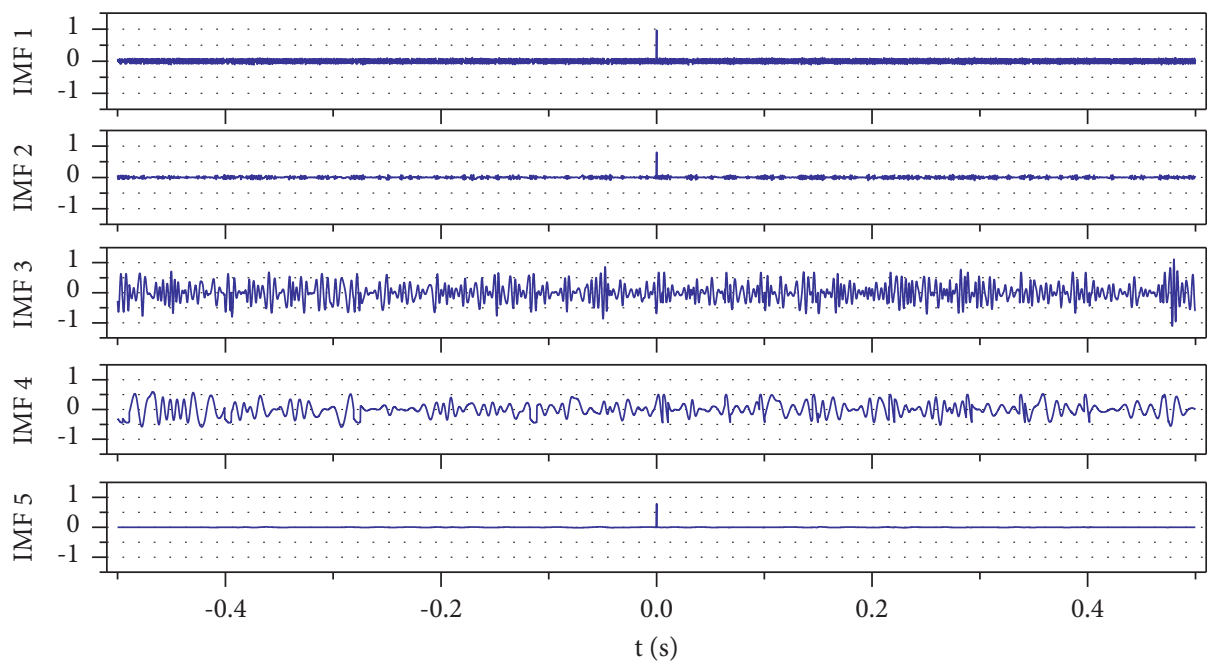

Figure 8: ACF of each IMF by EMD.

The similarity coefficient and the mean square error have been used to assess the degree of denoising performance of the mixed signal based on IEMD-IWFD and EMD.

The similarity coefficient is defined as

$$
\varepsilon_{i}=\frac{\left|\sum_{n=1}^{N} s_{i}(n) y_{i}(n)\right|}{\sqrt{\sum_{n=1}^{N} s_{i}^{2}(n) \sum_{n=1}^{N} y_{i}^{2}(n)}}
$$

where $s(n)$ represents the target signal, i.e., the reference value, and $y(n)$ represents the restricted signal, i.e., the comparison value.

The mean square error of signal is defined as

$$
\sigma_{i}=\sqrt{\frac{1}{N} \sum_{n=1}^{N}\left|z_{i}(n)-s_{i}(n)\right|^{2}} .
$$

The similarity coefficient, mean square error, and timeconsuming between the reconstructed signal based on IEMD-IWFDM and EMD and the target signal are enumerated in Table 1 . The similarity coefficient and mean square error for the IEMD-IWFDM are higher and lower compared to those for the EMD, respectively, which reveals that the target signal can be effectively separated from the building vibration signal corrupted by noise based on the 


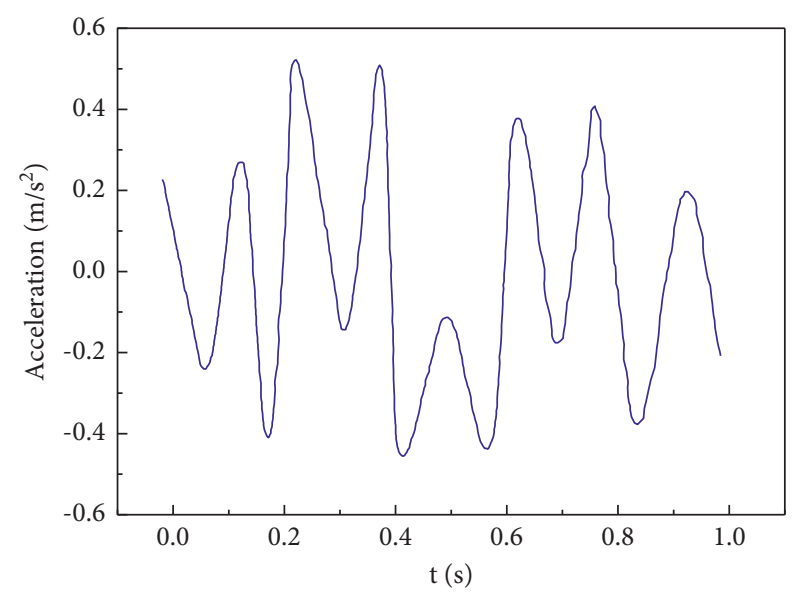

(a)

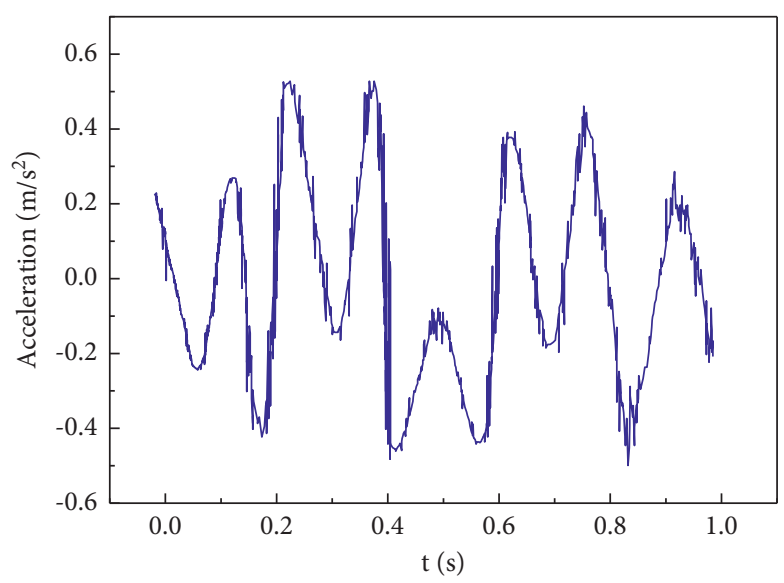

(b)

FIGURE 9: Reconstructed building vibration signal based on decomposed IMFs by IEMD-IWFD and EMD.

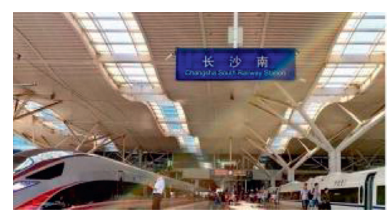

High-speed rail station

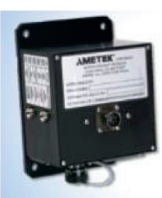

Accelerometer Sound level meter

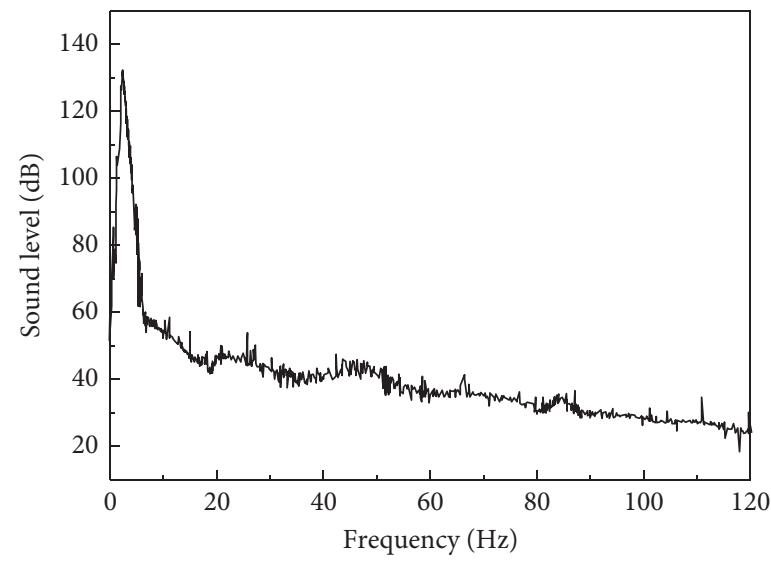

FiguRE 10: Building vibration signal at a high-speed rail station

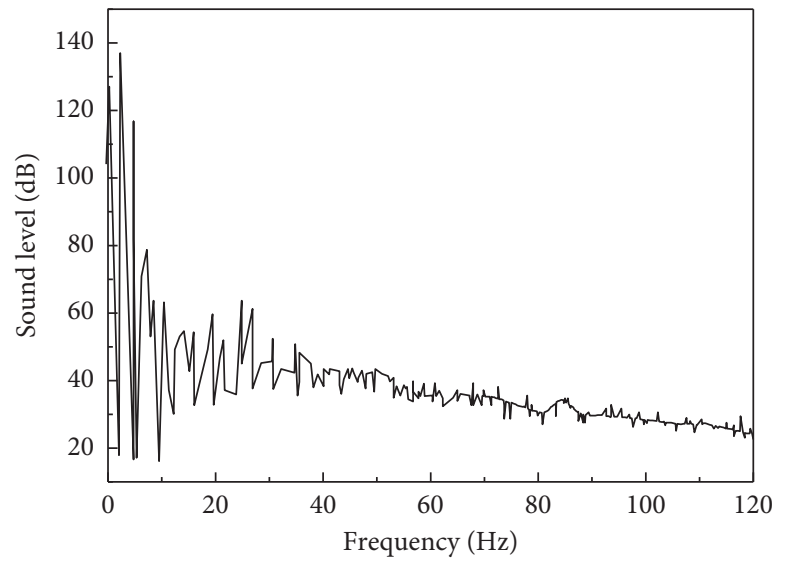

(a)

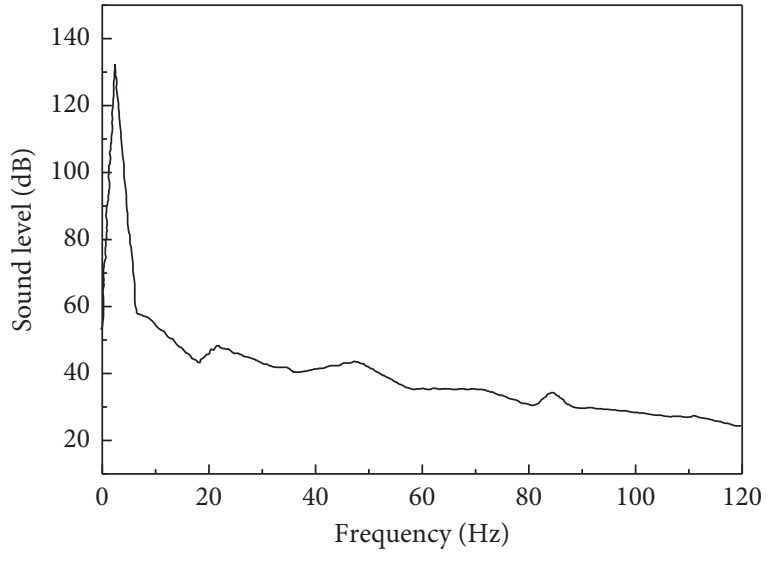

(b)

FIGURE 11: Denoised building vibration signal by EMD and IEMD-IWFD methods.

IEMD-IWFD. The time-consuming performances of EMD and IEMD-IWFDM methods are compared for computational complexity assessment. Obviously, the total CPU time of those methods depends on "the number of extrema points, the length of the signal, the number of IMFs decomposed, and other system setup expenses," which are 
TABLE 1: Evaluation of denoising performance for real building vibration signal.

\begin{tabular}{lcc}
\hline Parameters & IEMD-IWFDM & EMD \\
\hline Mean square error & 0.06 & 0.26 \\
Similarity coefficient & 0.97 & 0.62 \\
Times (s) & 0.28 & 0.23 \\
\hline
\end{tabular}

set as the same value to keep the comparability in the simulation. It can be seen from Table 1 that the IEMDIWFDM is slightly slower than the EMD, but it can achieve a more complete decomposition than the EMD.

\section{Conclusion}

In this study, a novel signal decomposition method was proposed by coupling the algorithms of IEMD and IWFD, which can be used in the denoising of building vibration signals. A synthetic building vibration was simulated by the combination of a target building vibration signal and a noise signal. It was first decomposed into some IMF components using the novel IEMD-IWFD and traditional EMD methods. The energy criterion of ACF was then employed to determine the IMFs dominated by useful information. Subsequently, the weight coefficient of each useful IMF was obtained by solving the coefficient matrix through the Hilbert transform. And the building vibration signal denoising was finally carried out by reconstructing the useful IMFs. The denoising effects for the IEMD-IWFD and EMD methods were compared by calculating the mean square error and similarity coefficient. The findings portrayed that the new method (IEMD-IWFD) can effectively extract the target signal from the signal corrupted by noise and achieve a better building vibration signal denoising performance. Next, we can make use of these clean signals to carry out statistical classification of feature information extracted from different types and scales and to realize multisource and multiparameter diagnoses of building vibration signals.

\section{Data Availability}

All data used to support the findings of this study are included within the article.

\section{Conflicts of Interest}

The authors declare that there are no conflicts of interest regarding the publication of this study.

\section{Acknowledgments}

The present research was supported by National Natural Science Foundation of China (Grant no. 51478470).

\section{References}

[1] J. D. Poston, J. Schloemann, R. M. Buehrer, and P. A. Tarazaga, "Towards indoor localization of pedestrians via smart building vibration sensing," in Proceedings of the
2015 international conference on localization and GNSS (ICLGNSS), pp. 1-6, IEEE, Gothenburg, Sweden, June 2015.

[2] C. Zou, Y. Wang, P. Wang, and J. Guo, "Measurement of ground and nearby building vibration and noise induced by trains in a metro depot," The Science of the Total Environment, vol. 536, pp. 761-773, 2015.

[3] Y. Lei, J. Lu, J. Huang, and S. Chen, "A general synthesis of identification and vibration control of building structures under unknown excitations," Mechanical Systems and Signal Processing, vol. 143, p. 106803, 2020.

[4] Q. He, X. Wang, and Q. Zhou, "Vibration sensor data denoising using a time-frequency manifold for machinery fault diagnosis," Sensors, vol. 14, pp. 382-402, 2014.

[5] S. Braun, "The synchronous (time domain) average revisited," Mechanical Systems and Signal Processing, vol. 25, no. 4, pp. 1087-1102, 2011.

[6] J.-B. Poullet, D. M. Sima, and S. Van Huffel, "MRS signal quantitation: a review of time- and frequency-domain methods," Journal of Magnetic Resonance, vol. 195, no. 2, pp. 134-144, 2008.

[7] M. S. McMullen and A. Jameson, "The computational efficiency of non-linear frequency domain methods," Journal of Computational Physics, vol. 212, no. 2, pp. 637-661, 2006.

[8] E. Sejdić, K. A. Lowry, J. Bellanca, M. S. Redfern, and J. S. Brach, "A comprehensive assessment of gait accelerometry signals in time, frequency and time-frequency domains," IEEE Transactions on Neural Systems and Rehabilitation Engineering, vol. 22, pp. 603-612, 2013.

[9] S. N. Chegini, A. Bagheri, and F. Najafi, "Application of a new EWT-based denoising technique in bearing fault diagnosis," Measurement, vol. 144, pp. 275-297, 2019.

[10] S. Hou, M. Liang, and Y. Li, "An optimal global projection denoising algorithm and its application to shaft orbit purification," Structural Health Monitoring, vol. 10, no. 6, pp. 603-616, 2011.

[11] G. Fan, J. Li, and H. Hao, "Vibration signal denoising for structural health monitoring by residual convolutional neural networks," Measurement, vol. 157, p. 107651, 2020.

[12] M. Zhao and X. Jia, "A novel strategy for signal denoising using reweighted SVD and its applications to weak fault feature enhancement of rotating machinery," Mechanical Systems and Signal Processing, vol. 94, pp. 129-147, 2017.

[13] D. S. Singh and Q. Zhao, "Pseudo-fault signal assisted EMD for fault detection and isolation in rotating machines," $M e$ chanical Systems and Signal Processing, vol. 81, pp. 202-218, 2016.

[14] J.-H. Ahn, D.-H. Kwak, and B.-H. Koh, "Fault detection of a roller-bearing system through the EMD of a wavelet denoised signal," Sensors, vol. 14, no. 8, pp. 15022-15038, 2014.

[15] M. Liu, K. Wang, L. Sun, and J. Zhen, "Applying empirical mode decomposition (EMD) and entropy to diagnose circuit breaker faults," Optik, vol. 126, pp. 2338-2342, 2015.

[16] R. Sharma and S. R. Mahadeva Prasanna, "A better decomposition of speech obtained using modified empirical mode decomposition," Digital Signal Processing, vol. 58, pp. 26-39, 2016.

[17] O. Niang, É. Delechelle, and J. Lemoine, “A spectral approach for sifting process in empirical mode decomposition," IEEE Transactions on Signal Processing, vol. 58, no. 11, pp. 56125623, 2010.

[18] Y. B. Li, M. Q. Xu, Y. Wei, and W. H. Huang, "Diagnostics of reciprocating compressor fault based on a new envelope algorithm of empirical mode decomposition," Journal of Vibroengineering, vol. 16, pp. 2269-2286, 2014. 
[19] H. Li, L. Li, and D. Zhao, "An improved EMD method with modified envelope algorithm based on $\mathrm{C} 2$ piecewise rational cubic spline interpolation for EMI signal decomposition," Applied Mathematics and Computation, vol. 335, pp. 112-123, 2018.

[20] J. Gilles, "Empirical wavelet transform," IEEE Transactions on Signal Processing, vol. 61, pp. 3999-4010, 2013.

[21] B. Boashash and S. Ouelha, "Efficient software platform TFSAP 7.1 and Matlab package to compute Time-Frequency Distributions and related Time-Scale methods with extraction of signal characteristics," Software, vol. 8, pp. 48-52, 2018.

[22] H. Li, L. Li, D. Zhao, J. Chen, and P. Wang, "Reconstruction and basis function construction of electromagnetic interference source signals based on Toeplitz-based singular value decomposition," IET Signal Processing, vol. 11, pp. 59-65, 2016.

[23] M. Salgarello, G. Visconti, and L. Barone-Adesi, "Interlocking circumareolar suture with undyed polyamide thread: a personal experience," Aesthetic Plastic Surgery, vol. 37, pp. 1061-1062, 2013.

[24] H. Ji, J. Long, Y. Fu, Z. Huang, B. Wang, and H. Li, "Flow pattern identification based on EMD and LS-SVM for gas-liquid two-phase flow in a minichannel," IEEE Transactions on Instrumentation and Measurement, vol. 60, pp. 1917-1924, 2011.

[25] S. A. Abdul Karim and K. Voon Pang, "Shape preserving interpolation using rational cubic spline," Journal of Applied Mathematics, vol. 8, no. 2, pp. 167-178, 2016.

[26] M. Abbas, A. A. Majid, and J. M. Ali, "Monotonicity-preserving C2 rational cubic spline for monotone data," Applied Mathematics and Computation, vol. 219, pp. 2885-2895, 2012.

[27] P. Lamberti and C. Manni, "Shape-preserving C2 functional interpolation via parametric cubics," Numerical Algorithms, vol. 28, pp. 229-254, 2001.

[28] R. G. Andrzejak, K. Schindler, and C. Rummel, "Nonrandomness, nonlinear dependence, and nonstationarity of electroencephalographic recordings from epilepsy patients," Physical Review, vol. 86, p. 046206, 2012.

[29] P. Singh, S. D. Joshi, R. K. Patney, and K. Saha, "The Fourier decomposition method for nonlinear and non-stationary time series analysis," Proceedings of the Royal Society A: Mathematical, Physical \& Engineering Sciences, vol. 473, p. 20160871, 2017.

[30] F. Rossi and A. Nicolini, "A simple model to predict traininduced vibration: theoretical formulation and experimental validation," Environmental Impact Assessment Review, vol. 23, pp. 305-329, 2003.

[31] P. Nassiri, M. Abbaspour, M. Mahmoodi, and S. Givargis, "A rail noise prediction model for the Tehran-Karaj commuter train," Applied Acoustics, vol. 68, pp. 326-333, 2007. 